Vegetatio 4: 182-194.

DEBAZAC, E.F. -1958- La végétation forestière de la Kroumirie. Ann. Ecol. Nat. Eaux For. Stat. Rech. Exp. 16(1): 1-133.

FINCKH, M. \& U. DEIL -1989- Approches de l'étude de la degradation des fôrets du Jbel Bougriy (Moyen Atlas Central). RGM (n.s.) 13(1): 55-68.

LADERO, M., E. BIONDI, L. MOSSA y A. AMOR -1992-Los pastizales mediterráneos presididos por Trifolium slibterraneum en la isla de Cerdeña (Italia). Doc. Phytosociol. (n.s.) 14: 45-63.

NÈGRE, R. -1961- Les associations végétales du Jbel Saa. Bull. Soc. Sci. Nat. Maroc 41: 19-62.

RIVAS GODAY, S. y S. RIVAS-MARTÍNEZ 1963-Estudio y clasificación de los pastizales españoles. Publ. Ministerio de Agricultura. Madrid.

RIVAS GODAY, S. -1964-Vegetación y flórula de la cuenca extremeña del Guadiana. Publ. Dip. Prov. Badajoz. Madrid.
RIVAS GODAY, S. y M. LADERO ÁLVAREZ 1970- Pastizales cespitosos de Poa bulbosa L. Origen, sucesión y sistemática. Anales R. Acad. Farmacia 36(2): 139-181.

RIVAS-MARTíNEZ, S. -1978-Sur la syntaxonomie des pelouses therophytiques de l'Europe occidentale. Coll. Phytosoc. 6: 55-71.

RIVAS-MARTÍNEZ, S., F. FERNÁNDEZ GONZÁLEZ y D. SÁNCHEZ-MATA -1986Datos sobre la vegetación del Sistema Central y Sierra Nevada. Opusc. Bot. Pharm. Complutensis 2: 1-136.

Aceptado para su publicación en Marzo de 1997

Dirección de los autores. Departamento de Biología. Laboratorio de Botánica. Universidad San PabloCEU. Ap. 67. E-28660 Boadilla del Monte (Madrid). Fax. (91) 3510475 .

\title{
65. ASPECTOS NOMENCLATURALES, ECOLÓGICOS Y BIOGEOGRÁFICOS SOBRE LA RESEDETUM VALENTINAE (ALL. SCROPHULARION SCIOPHILAE)
}

\author{
Manuel B. CRESPO, José Luis SOLANAS, Ana JUAN y Luis SERRA.
}

Nomenclatural, ecological and biogeographical features about Resedetum valentinae (All. Scrophularion sciophilae)

Palabras clave. Sintaxonomía, fitosociología, nomenclatura, Scrophularion sciophilae, Alicante, España.

Key words. Syntaxonomy, phytosociology, nomenclature, Scrophularion sciophilae, Alicante, Spain.

En el contexto de una revisión de las comunidades glareícolas iberolevantinas, $\mathrm{O}$. Bolòs (1974) describió la asociación Resedetum valentinae - Scrophularion sciophilae $\mathrm{O}$. Bolòs 1957, Andryaletalia ragusinae Rivas
Goday \& Rivas Mart. ex O. Bolòs \& Vigo in Folch 1981, Thlaspietea rotundifolii Br.-Bl. 1947 - sobre la base de un inventario tomado en las proximidades de la Font Roja de Alcoi (Alicante), territorio incluido en el subsector 
Alcoyano-Diánico (De la Torre et al., 1996) del sector Setabense (provincia CatalanoValenciano-Provenzal). En la diagnosis original se caracterizaba implícitamente dicha asociación en los siguientes términos: "El Scrophulario-Arenarietum intricatae de los pedregales calizos de las montañas valencianas meridionales es reemplazado, al parecer, en algunas áreas montañosas interiores por otra comunidad en la que destaca Reseda alba subsp. alba var. valentina".

El sintaxon toma nombre del endemismo de óptimo setabense Reseda valentina (Pau) Pau ex Cámara subsp. valentina, binomen que, como ha demostrado Crespo (1993) y pese a lo indicado por Valdés-Bermejo \& Kaercher (1984) y Valdés-Bermejo (1993), debe considerarse legítimo y obviamente prioritario frente a $R$. paui Valdés-Bermejo \& Kaercher. Sin embargo, la asociación fue descrita explícitamente como provisional y por tanto en el momento y lugar de su publicación original (O. Bolòs, 1974: 78) no podía considerarse válidamente publicada.

Posteriormente, Folch (1981: 414) incluyó la Resedetum valentinae en el cuadro sintaxonómico de las comunidades presentes en los territorios iberolevantinos, considerando como fecha y lugar de publicación efectiva el citado trabajo de O. Bolòs de 1974 y caracterizando la asociación en el mismo sentido que hiciera aquél, aunque precisando (op. cit.: 190) que la asociación se desarrolla en las comarcas alicantinas interiores (l'Alcoià, etc.). Por tanto, Folch validó indirectamente la Resedetum valentinae al aceptarla como buena asociación y al satisfacer la única condición que hasta entonces invalidaba el nombre del sintaxon (Art. 6 del Código de Nomenclatura Fitosociológica; Izco \& Del Arco-Aguilar, 1988).

Respecto a la tipificación de la asociación debe tenerse en cuenta lo indicado en el artículo 5. La validación de nombre del sintaxon se realizó con posterioridad al 1 -enero-1979, sin hacer mención explícita de su tipo nomenclatural. No obstante, dado que en la diagnosis original de la Resedetum valentinae se adjuntó un solo inventario, éste debe considerarse automáticamente holotipo (Art. 18). Pese a que dicho inventario no es un fiel exponente de la asociación tal y como la conocemos en toda su área, lógicamente no puede completarse ni modificarse (Art. 16). Por otra parte, y aunque el holotipo pueda considerarse incompleto, la caracterización ecológica y corológica de la asociación ofrecida en la diagnosis original no deja lugar a dudas sobre la aplicación del nombre, siendo improcedente su eventual corrección o rechazo (Arts. 29, 37).

Más recientemente, Stübing et al. (1992: 182) han propuesto la Scrophulario sciophilaeIberidetum hegelmaieri, asociación que consideran distribuida óptimamente por las áreas mesomediterráneas subhúmedas del subsector Alcoyano-Diánico y cuya localidad clásica (tab. 2, inv. 2) se sitúa en el "Carrascal de Alcoy, Alicante". Aunque en la tabla original de esta asociación no aparece Reseda valentina, según hemos podido constatar sobre el terreno dicha planta aparece con gran constancia en las comunidades glareícolas alicantinas y valencianas; aunque no resulta exclusiva de dichos hábitats, ya que también participa en asociaciones de Asplenietea trichomanis. En nuestra opinión existen razones florísticas, ecológicas, corológicas y bioclimáticas suficientes para considerar ambas asociaciones sinónimas. Por otra parte, no parece necesario proponer enmiendas a la asociación, dado que tal proceder no queda tipificado ex plícitamente en el C.N.F. A este respecto, los ejemplos de enmiendas recogidos en el Código afectan a los sintaxones de rango superior a la asociación y, además, la estricta aplicación de la recomendación $47 \mathrm{~A}$-que regula las modificaciones en la delimitación de los sintaxones-podría conducir a la enmienda de cualquier sintaxon de rango igual o inferior a 
la asociación, lo cual no es deseable en aras a la estabilidad nomenclatural.

Según lo expuesto anteriormente la citación correcta, tipo nomenclatural y sinónimos de la asociación son los siguientes:

Resedetum valentinae $\mathrm{O}$. Bolòs ex Folch 1981 [La vegetació dels Països Catalans: 414. Holotypus: O. Bolòs, Miscell. Alcobé: 78. 1974]

Syn.: Scrophulario sciophilae-Iberidetum hegelmaieri Stübing et al. 1992, Fol. Bot. Misc. 8: 182 [Holotypus: tabla 2, inv. 2.]

Taxones característicos: Reseda valentina (Pau) Pau ex Cámara, Scrophularia sciophila Willk. (all.), Iberis carnosa Willd. subsp. hegelmaieri (Willk.) Moreno, Linaria depauperata Leresche ex Lange, Pimpinella gracilis (Boiss.) Pau (terr.), Saponaria ocymoides L. (terr.).

Ecología y bioclimatología: Canchales y taludes pedregosos calizos, en áreas (supra-) mesomediterráneas de matiz continental, bajo ombroclima subhúmedo (más raramente seco).

Distribución: Óptimo en el sector Setabense (subsectores Alcoyano-Diánico, Enguerino-Cofrentino y Ayorano-Villenense), aunque también penetra ligeramente en los territorios colindantes.

\section{BIBLIOGRAFÍA}

BOLÒs, O. -1974- Notas sobre vegetación glareícola. Miscellanea Alcobé 77-86.

CRESPO, M.B. -1993-Reseda valentina (Resedaceae) - a legitimate name. Willdenowia 23: $103-106$.

DE LA TORRE, A., F. ALCARAZ y M.B. CRESPO -1996- Aproximación a la biogeografía del sector Setabense (provincia CatalanoValenciano-Provenzal). Lazaroa 16: 141-158.

FOLCH, R. -1981- La vegetació dels Països Catalans. Ketres. Barcelona.
IZCO, J. y M.J. DEL ARCO-AGUILAR -1988Código de nomenclatura fitosociológica. Opusc. Bot. Pharm. Complutensis 4: 5-74.

STÜBING, G., J.B. PERIS, R. FIGUEROLA, P. BALLESTER y F. ESTESO -1992- La alianza Scrophularion sciophilae en el territorio valenciano. Fol. Bot. Miscel. 8: 181-187.

VALDÉS-BERMEJO, E. -1993- Reseda L. In: CASTROVIEJO, S. et al. (eds.), Flora iberica 4: 440-475. Real Jardín Botánico, CSIC. Madrid. VALDÉS-BERMEJO, E. y W. KAERCHER -1984Dos nuevos taxones ibéricos del género Reseda L. sect. Leucoreseda DC. Anales Jard. Bot. Madrid 41: 198-201.

Aceptado para su publicación en Enero de 1997

Dirección de los autores. Departamento de Ciencias Ambientales y Recursos Naturales (Botánica). Universidad de Alicante. Apartado 99. E-03080 Alicante. 